\title{
Mercurio total en hígado de Trachemys callirostris (Gray, 1856) (Testudines: Emydidae) en tres zonas de la Mojana, Sucre-Colombia
}

\section{Total mercury in liver of Trachemys callirostris (Gray, 1856) (Testudines: Emydidae) in three areas of la Mojana, Sucre-Colombia}

Mayra Meza-Martínez¹; Jaime De La Ossa-Velázquez²; Jesús Hernández-Gómez³; José Marrugo-Negrete ${ }^{4}$

'Bióloga, M.Sc. Universidad de Sucre. Maestría en Ciencias Ambientales. Sincelejo - Sucre, Colombia; e-mail: mairamezamartinez@gmail.com; (D) https://orcid.org/0000-0002-6150-1314

Lic. en Biología, Ph.D. Universidad de Sucre. Grupo de Investigación en Biodiversidad Tropical. Sincelejo - Sucre, Colombia; e-mail: jaime.delaossa@ unisucre.edu.co; (1) https://orcid.org/0000-0002-9374-7765

${ }^{3}$ Biólogo. Universidad de Sucre. Sincelejo - Sucre, Colombia; e-mail: jesus7hdez@gmail.com; (D) https://orcid.org/0000-0002-4298-0292

${ }^{4}$ Ing. Químico, Ph.D. Universidad de Córdoba. Grupo de Aguas, Química Aplicada y Ambiental, Laboratorio de Toxicología y Gestión Ambiental. Montería Córdoba, Colombia; e-mail: jmarrugo@correo.unicordoba.edu.co; (D) https://orcid.org/0000-0002-3181-7529

Cómo citar: Meza-Martínez, M.; De La Ossa-Velázquez, J.; Hernández-Gómez, J.; Marrugo-Negrete, J. 2020. Mercurio total en hígado de Trachemys callirostris (Gray, 1856) (Testudines: Emydidae) en tres zonas de la Mojana, Sucre-Colombia. Rev. U.D.C.A Act. \& Div. Cient. 23(1):e1239. http://doi.org/10.31910/rudca.v23.n1.2020.1239

Artículo de acceso abierto publicado por Revista U.D.C.A Actualidad \& Divulgación Científica, bajo una licencia Creative Commons CC BY-NC 4.0

Publicación oficial de la Universidad de Ciencias Aplicadas y Ambientales U.D.C.A, Institución de Educación Superior Acreditada de Alta Calidad por el Ministerio de Educación Nacional.

Recibido: Junio 18 de 2019

Aceptado: Abril 2 de 2020

Editado por: Ingeborg Zenner de Polanía

\section{RESUMEN}

La región de la Mojana, perteneciente a la depresión Momposina, que se encuentra en el norte colombiano, desde hace muchos años, ha estado presentando problemáticas ambientales, asociadas a los metales pesados, en principio, por mercurio que, potencialmente, está afectando la fauna silvestre, en especial, a Trachemys callirostris. Esta especie categorizada como vulnerable, se encuentra amenazada, esencialmente, por la sobreexplotación y la alteración de su hábitat. El objetivo de este estudio fue determinar las concentraciones de mercurio total en hígado de la hicotea T. callirostris en tres localidades del departamento de Sucre, pertenecientes a la región de la Mojana. Los individuos, se obtuvieron, de manera directa, de pescadores de subsistencia, en los municipios de San Marcos, Caimito y Guaranda. Se tomaron muestras de hígado de 35 individuos de T. callirostris y se cuantificaron las concentraciones de mercurio total, por el método de Espectrometría de Absorción Atómica, usando un analizador directo de mercurio, con previos tratamientos de las muestras. Los resultados mostraron diferencias significativas entre los sitios muestreados $(\mathrm{p}<0,05)$ en las concentraciones de mercurio. Las concentraciones de mercurio encontradas en este estudio 
demuestran el potencial riesgo ambiental para las especies que comparten este hábitat, en especial, las de consumo humano.

Palabras clave: metales pesados; contaminación; Cheloniidae; minería; bioacumulación.

\section{ABSTRACT}

The Mojana region, belonging to the Momposina depression in northern Colombia, presents environmental problems associated with heavy metals for many years, mainly due to mercury, which is potentially affecting wildlife, especially Trachemys callirostris. This species categorized as vulnerable, is mainly threatened by overexploitation and alteration of its habitat. The objective of this study was to determine the concentrations of total mercury in the liver of the hicotea T. callirostris in three locations in the department of Sucre belonging to the Mojana region. Turtles were obtained directly from subsistence fishermen from towns San Marcos, Caimito and Guaranda. Liver samples were taken from 35 individuals of T. callirostris and total mercury concentrations were also quantified by the Atomic Absorption Spectrometry method, using a mercury direct analyzer with previous sample treatments. The results showed significant differences between the sampled sites $(p<0.05)$ in mercury concentrations. The mercury concentrations found in this study demonstrate the potential environmental risk for the species that share this habitat, especially those for human consumption.

Keywords: Heavy metals; Cheloniidae; contamination; mining; bioaccumulation.

\section{INTRODUCCIÓN}

A raíz de la actividad aurífera, Colombia se ha convertido en uno de los países que más contamina con mercurio al medio ambiente (Marrugo-Negrete et al. 2020); en los últimos años, ha aumentado de manera considerable la extracción de oro, de manera artesanal y a pequeña escala, principalmente, en aquellas regiones, en las que, por tradición, se ha extraído este metal, donde la mayoría de esas actividades mineras son ilegales (Diaz et al. 2020). Existe una preocupación creciente sobre los efectos nocivos de la contaminación por mercurio sobre la población humana (Marrugo et al. 2018; Rocha-Román et al. 2018) y la fauna de los diversos ecosistemas involucrados (Cruz-Esquivel et al. 2017; MarrugoNegrete et al. 2019).

La Mojana, una subregión al norte de Colombia, que hace parte de la Depresión Momposina y cumple la labor de regular los cauces de los ríos Magdalena, Cauca y San Jorge, se ha visto afectada por la extracción de oro, desde hace mucho tiempo (Parra et al. 2019). Una de las especies silvestres que habita esa zona es la tortuga Trachemys callirostris o Trachemys ornata callirostris, sensu (Fritz et al. 2012), que en el país se distribuye en la región Caribe, desde el Golfo de Urabá hasta el Sur de La Guajira, incluyendo los complejos cenagosos del Bajo río Sinú, Bajo Cauca, San Jorge, Magdalena y Ranchería (Rueda-Almonacid et al. 2007; Bock et al. 2010; Páez et al. 2012).

T. callirostris, se caracteriza por ser longeva y por la gran capacidad de bioacumular mercurio (Bergeron et al. 2010; Zapata et al. 2014), debido a su dieta omnívora, tener una posición entre la mitad y la cima de la cadena trófica y por habitar áreas contaminadas (Zapata et al. 2016). A pesar de ser ilegal en Colombia su extracción intensiva y su comercialización, T. callirostris está siendo sobreexplotada para la venta y consumo humano. Entre los usos que se le da a esta especie resaltan desde el consumo de carne y huevos hasta su uso medicinal. Esta situación, la convierte en una especie vulnerable, lo que pone en riesgo su conservación (De La Ossa-V \& De La Ossa-Lacayo, 2018). Además de esto, se le suma la pérdida y la degradación del hábitat, principalmente, por los impactos generados en la minería aurífera (Leguizamo \& Bonilla, 2014).

Existen estudios en los que se ha demostrado que los quelonios pueden ser usados como biocentinelas para la detección de contaminación por metales pesados, tales como el mercurio en los ecosistemas que habitan; por ejemplo, altas concentraciones de mercurio han sido reportadas en hígado de Podocnemis unifilis y Chelonia mydas y niveles más bajos, en músculo, sangre, riñón y tejido graso (Bezerra et al. 2013; Souza-Araujo et al. 2015). También, Green et al. (2010) evaluaron las concentraciones de mercurio en diferentes tejidos para 14 especies de tortugas, siendo el tejido hepático, el que mostró las concentraciones más altas para este metal.

A raíz de ello, los metales pesados pueden actuar como genotóxicos, provocando alteraciones genéticas en la integridad y el funcionamiento del ADN de diferentes especies animales y, por lo tanto, la evidencia del daño genético es un biomarcador muy importante, que se usa como una herramienta para la prevención de poblaciones en riesgo (Bionda et al. 2012; Cruz-Esquivel et al. 2017). Se ha evaluado la genotoxicidad ocasionada por algunos contaminantes en diferentes especies animales, utilizando la prueba de micronúcleos, la cual, ha puesto en evidencia el daño genético que ha producido en ellas, por las actividades antrópicas, tales como el vertimiento de residuos de actividades agrícolas y mineras, a diversos cuerpos de agua (Zapata et al. 2016; Cruz-Esquivel et al. 2017).

A pesar que en la Mojana sucreña no existen actividades de minería aurífera, se pueden encontrar concentraciones considerables de mercurio, en los diferentes compartimentos ambientales, gracias a que, tanto en la cuenca del río San Jorge como en la cuenca del río Cauca, se desarrollan este tipo de actividades mineras, donde se generan grandes cantidades de desechos altamente tóxicos, que quedan depositados, por vertimientos a los cuerpos de agua, favoreciendo el transporte de estos contaminantes aguas abajo de los ríos, desde los sitios mineros vecinos, en el sur de Bolívar, Antioquia y Córdoba(CORANTIOQUIA, 2008; MINMINAS, 2014).

Por todo lo anterior, el objetivo de este estudio fue determinar las concentraciones de mercurio total en hígado de T. callirostris, en tres zonas de la Mojana sucreña. 


\section{MATERIALES Y MÉTODOS}

Área de estudio. Se escogieron tres municipios ribereños localizados antes de las desembocaduras de los ríos Cauca y San Jorge, en el departamento de Sucre, ubicados aguas abajo de importantes focos de minería aurífera del norte de Colombia: San Marcos, Caimito y Guaranda. Las zonas de procedencia de los individuos, reportadas por los pescadores, fueron 1) Complejo cenagoso de la

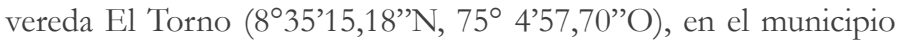
de San Marcos y 2) Complejo cenagoso de El Mamón y Pueblo Búho ( $8^{\circ} 45$ '2,53'N, $75^{\circ} 3^{\prime}$ '0,35'O), en el municipio de Caimito; estas dos localidades hacen parte de la cuenca del río San Jorge. 3) Ciénaga del Medio ( $8^{\circ} 28^{\prime} 45,20^{\prime}$ N $\left., 74^{\circ} 32^{\prime} 46,60^{\prime \prime} \mathrm{O}\right)$, en el municipio de Guaranda, ribereño del río Cauca (Figura 1); esta región del departamento, se caracteriza por pertenecer a la zona de vida Bosque Seco Tropical (Bs-T), según Holdridge (1967).
Colecta de muestras. El muestreo realizado fue por conveniencia en los tres sitios en la época de sequía, durante marzo y abril de 2018. Se obtuvieron un total de 35 individuos hembras (Caimito, $\mathrm{n}=13$; San Marcos, $\mathrm{n}=12$; Guaranda, $\mathrm{n}=10$ ). Se hicieron visitas a los tres municipios, donde la población, tradicionalmente, consume carne de hicotea, en esta época del año. Las tortugas, se obtuvieron en pequeños mercados locales, directamente de pescadores de subsistencia, quienes reportaron el sitio de procedencia de cada individuo. En este estudio, se escogieron solo los individuos que habían sido capturados utilizando lanzas; aquellos que no se encontraban en buen estado de salud, se descartaron. Todos los ejemplares fueron hembras adultas, ya que los cazadores las prefieren, debido a su gran tamaño, para la venta.

Muestras biológicas. A cada uno de los individuos, se le midió la Longitud Total del Caparax (LTC), utilizando un calibrador análogo

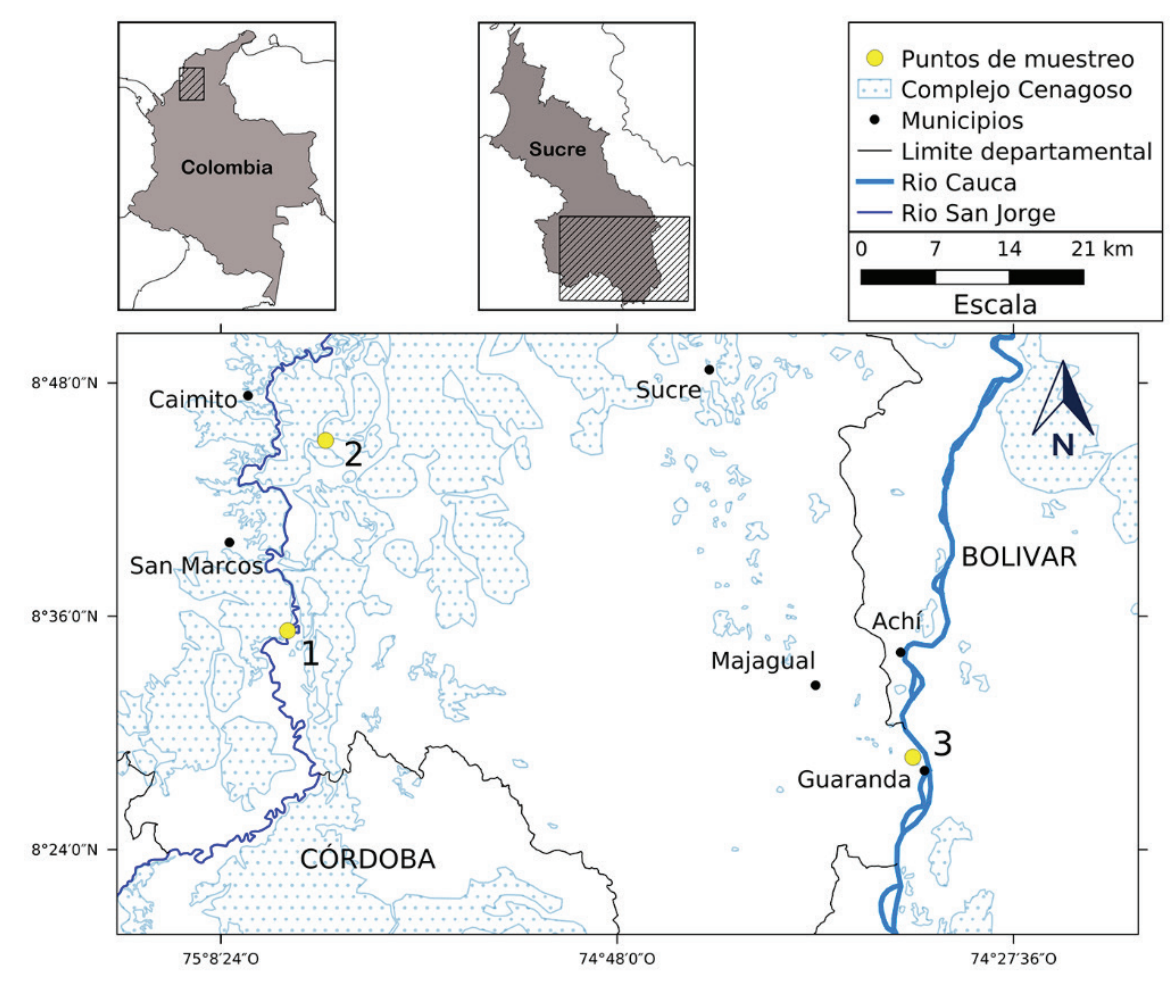

Figura 1. Zonas de muestreo seleccionadas en la Mojana sucreña. Mapa realizado en el programa QGIS 3.6.3 'Noosa'.

Mahr 12 pulgadas (0,02mm). El criterio de inclusión que se tuvo en cuenta fue que se tomaran solo los ejemplares que excedían el tamaño de la longitud total del caparax (LTC), en la que se consideran hembras maduras $(>15 \mathrm{~cm}$ ) (Bock et al. 2012).

Las muestras, se tomaron solo en los individuos que habían fallecido a causa del método de captura. Luego de romper el plastrón y exponer la región peritoneal, usando escalpelos y tijeras estériles, se recogieron muestras de aproximadamente, $5 \mathrm{~g}$ de hígado en cada individuo, para los análisis de mercurio (Melidone \& Gibson, 2010).
Detección de mercurio. Después de estandarizar los protocolos para el procesamiento de las muestras de hígado, se realizó la determinación de mercurio por espectrometría de absorción atómica, usando un analizador directo de mercurio (DMA80 TRICELL, Milestone Inc., Italy), posterior a la digestión ácida de la muestra extraída (Sadiq et al. 1991). Las concentraciones de mercurio total, se reportaron con base en su peso húmedo y se expresaron como THg, $\mu \mathrm{g} / \mathrm{kg}$ ww. 
Análisis estadístico. Se realizó la estadística descriptiva (media \pm desviación estándar) para las variables evaluadas (LTC y concentración de mercurio). Se realizó la prueba no paramétrica de Kruskal \& Wallis (1952), para determinar si existían diferencias significativas $(\mathrm{p}<0,05)$ entre los sitios estudiados, previa corroboración de la normalidad de los datos. Para comparar los sitios, se hizo la comparación de a pares, descrito por Conover (1999). Todas las pruebas fueron realizadas en el programa Infostat versión 2018 (Di Rienzo et al. 2011).

\section{RESULTADOS Y DISCUSIÓN}

Todos los ejemplares muestreados fueron hembras adultas, con presencia de huevos en su interior (Tabla 1).
Para la concentración de mercurio, los grupos de San Marcos y de Caimito presentaron mayores promedios de concentración con respecto al grupo de Guaranda $(\mathrm{p}<0,05)$ (Tabla 1). Valores similares han sido reportados en otros estudios en sedimentos de zonas inundadas por el río San Jorge, cerca de la hacienda Lorenzana (San Marcos), concentraciones que estaban entre los 3,4 y $632,7 \mu \mathrm{gHg} / \mathrm{kg}$ (CORANTIOQUIA, 2008). Las muestras de los individuos colectados en Guaranda presentaron un promedio bajo de concentración de mercurio en sangre, similares a los reportados por Marrugo-Negrete et al. (2019), de una especie de caiman, procedente de zonas inundadas por el río Cauca. Todos los promedios de las muestras son mucho mayores a los reportados en Melanosuchus niger y Podocnemis expansa, en músculo, en queratina y en hueso, en estas dos especies (Schneider et al. 2015).

Tabla 1. Media \pm desviación estándar de la longitud total del caparax (LTC, mm) y mercurio total en tejido hepático (THg, $\mu \mathrm{g} / \mathrm{kg}$ ww), de T. callirostris, en los tres sitios de estudio.

\begin{tabular}{|c|c|c|c|c|c|}
\hline Variables & $\begin{array}{c}\text { Caimito } \\
(\mathbf{n}=\mathbf{1 3})\end{array}$ & $\begin{array}{c}\text { Guaranda } \\
\mathbf{( n = 1 0 )}\end{array}$ & $\begin{array}{c}\text { San Marcos } \\
\mathbf{( n = 1 0 )}\end{array}$ & Mínimo-Máximo & p valor \\
\hline LTC & $230,55 \pm 8,52^{\mathrm{a}}$ & $229,19 \pm 12,29^{\mathrm{a}}$ & $236,31 \pm 15,35^{\mathrm{a}}$ & $210,12-266,60$ & 0,4042 \\
\hline $\mathrm{Hg}$ & $113,55 \pm 43,55^{\mathrm{b}}$ & $69,87 \pm 25,15^{\mathrm{a}}$ & $166,13 \pm 71,27^{\mathrm{b}}$ & $38,54-278,06$ & 0,0011 \\
\hline
\end{tabular}

LTC: Longitud Total del Caparax.

Letras minúsculas diferentes indican diferencias significativas entre zonas para cada variable $(\mathrm{p}<0,05)$.

Los valores de Hg en tejidos de hicotea, también han sido evaluados

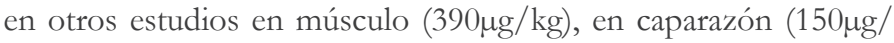
$\mathrm{kg}$ ) y en sangre $(70 \mu \mathrm{g} / \mathrm{kg}$ ) (Zapata et al. 2014). En otras especies de reptiles del orden Crocodilia, también se ha evaluado este metal pesado en diferentes tejidos; para la especie Caiman crocodilus, se reportaron concentraciones de mercurio en garras, placas o escamas y sangre $(1100 \mu \mathrm{g} / \mathrm{kg}, 490 \mu \mathrm{g} / \mathrm{kg}$ y $65 \mu \mathrm{g} / \mathrm{kg}$, respectivamente), siendo este último tejido, con la menor concentración del metal (MarrugoNegrete et al. 2019). Los valores anteriormente descritos están cercanos a los reportados en este estudio. La mayor concentración de mercurio total encontrada en el presente estudio fue de 278,06 $\mu \mathrm{g} / \mathrm{kg}$ ww, alcanzada por un individuo proveniente de San Marcos.

La presencia de mercurio en tejido hepático en este estudio, se debe a que este metal se puede bioacumular en varios tejidos de los organismos, a diferentes concentraciones. Este contaminante es altamente soluble en lípidos y se puede encontrar en mayor concentración en tejido hepático, debido a que el metilmercurio, al ingresar al organismo, puede formar enlaces covalentes con los grupos sulfhidrilo de las enzimas, por esto, es que se va acumulando en tejidos con alto contenido de proteínas, como el hígado (Hong et al. 2012; Arantes et al. 2016). Estos órganos están expuestos a este contaminante por varias vías, que le permiten absorberlo, a través de la ingestión de agua y sedimentos de su entorno, por inhalación de aire contaminado, transferencia hacia la descendencia y del medio en que se encuentren los nidos (Beyer \& Fries, 2002; Smith et al. 2007).
Las concentraciones medias de mercurio total documentadas en hígado de T. callirostris en este estudio, no excedieron el nivel máximo $(500 \mu \mathrm{g} / \mathrm{kg})$, recomendado por la Organización Mundial de la Salud, en especies de peces consumidas por humanos (FAO, 2015). Algunas investigaciones han reportado los niveles de exposición a los que puede estar sometida esta especie. Estos estudios han hallado concentraciones más altas que las encontradas en esta investigación. Páez-Osuna et al. (2011), en la tortuga olivácea o golfina, Lepidochelys olivacea, encontraron que los niveles de $\mathrm{Hg}$ estaban por debajo de las pautas establecidas de seguridad alimentaria de varias naciones (FAO, 2015); asimismo, Green et al. (2010), en un estudio con diferentes especies de tortugas, reportaron altos niveles de mercurio en los organismos carnívoros con respecto a los herbívoros y de los diferentes tejidos analizados; en hígado, se reportaron los más altos niveles, para todas las especies.

En otras especies, como animales domésticos utilizados para el consumo humano, han sido reportadas altas concentraciones de mercurio en zonas con actividades mineras; se encontraron en las especies Sus scrofa $(8156,9 \mu \mathrm{g} / \mathrm{kg})$, Gallus gallus $(3391,9 \mu \mathrm{g} / \mathrm{kg})$ y Cairina moschata $(1426,5 \mu \mathrm{g} / \mathrm{kg})$, representando un alto riesgo para la salud pública (Argumedo et al. 2013).

Existen estudios que han demostrado que los metales pesados, al ingresar al organismo, son capaces de envenenar la función celular, perjudicando la función normal de cualquier órgano o estructura 
subcelular, perturbando el buen desarrollo de los organismos y sus funciones fisiológicas (Afshan et al. 2014; Morcillo et al. 2016). Los hallazgos de este estudio demuestran bioconcentración en fauna silvestre, por la presencia de mercurio, lo que, a su vez, representa un riesgo ambiental para todas las especies que habitan en esta área, en particular, aquellas especies de mayor consumo humano. Por lo anterior, se recomienda evitar el consumo de carne de esta especie, debido a las concentraciones de mercurio en tejidos. También ampliar el número de localidades y de individuos por localidad, para evaluar los niveles de mercurio y otros contaminantes posibles de la zona, como plaguicidas. Así como implementar un grupo control negativo, aislado geográficamente, de zonas que presentan contaminación por metales pesados.

Agradecimientos: A la Universidad de Córdoba - Montería, Colombia, por la financiación de esta investigación. También, al laboratorio de toxicología y gestión ambiental de la Universidad de Córdoba, por el apoyo logístico en el procesamiento de las muestras. A la Corporación para el Desarrollo Sostenible de la Mojana y el San Jorge (CORPOMOJANA), por los permisos otorgados para la captura y el sacrificio de los individuos, para la colecta de las muestras biológicas. Finalmente, al Biólogo Ángel Cruz Esquivel, por su colaboración durante el desarrollo de este trabajo. Conflicto de intereses: Los autores manifestamos que no existen relaciones económicas o de otra índole que podrían conducir a un conflicto de intereses entre las partes.

\section{REFERENCIAS}

1. AFSHAN, S.; ALI, S.; AMEEN, U.S.; FARID, M.; BHARWANA, S.A.; HANNAN, F; AHMAD, R. 2014. Effect of different heavy metal pollution on fish. Research J. Chemical and Environmental Sciences (India). 2(1):74-79.

2. ARANTES, F.P.; SAVASSI, L.A.; SANTOS, H.B.; GOMES, M.V.; BAZZOLI, N. 2016. Bioaccumulation of mercury, cadmium, zinc, chromium, and lead in muscle, liver, and spleen tissues of a large commercially valuable catfish species from Brazil. Anais da Academia Brasileira de Ciências (Brasil). 88(1):137-147. http://doi.org/10.1590/00013765201620140434

3. ARGUMEDO, M.; VIDAL, J.; MARRUGO, J. 2013. Mercurio total en animales domésticos en mina Santa Cruz, Sur de Bolívar-Colombia. Rev. Colombiana de Ciencia Animal (Colombia). 5(2):366-379. https://doi.org/10.24188/recia. v5.n2.2013.449

4. BERGERON, C.M.; BODINOF, C.M.; UNRINE, J.M.; HOPKINS, W.A. 2010. Bioaccumulation and maternal transfer of mercury and selenium in amphibians. Environmental toxicology chemistry (Estados Unidos). 29(4):989-997. https://doi.org/10.1002/etc.125

5. BEYER, W.N.; FRIES, G.F. 2002. Toxicological significance of soil ingestion by wild and domestic animals. En: Hoffman,
D.J.; Rattner, B.A.; Burton, G.A.; Cairns, J. (eds). Handbook of Ecotoxicology, . CRC Press (Boca Raton, Florida). p.175-190. https://doi.org/10.1201/9781420032505

6. BEZERRA, M.F.; LACERDA, L.D.; LIMA, E.H.; MELO, M.T. 2013. Monitoring mercury in green sea turtles using keratinized carapace fragments (scutes). Marine Pollution Bulletin (Reino Unido). 77:424-427. https:/ / doi. org/10.1016/j.marpolbul.2013.09.020

7. BIONDA, C.; SALAS, N.; CARAFFA, E.; BARAQUET, M.; MARTINO, A. 2012. On abnormalities recorded in an urban population of Rhinella arenarum from central Argentina. Herpetology Notes (Europa). 5:237-241.

8. BOCK, B.; PÁEZ, V.; DAZA, J. 2010. Trachemys callirostris (Gray 1856)-Colombian slider, jicotea, hicotea, galapago, morrocoy de agua. En: Rhodin, A. G. J.; Pritchard, P. C. H.; van Dijk, P. P.; Saumure, R. A.; Buhlmann, K. A.; Iverson, J. B.; Mittermeier, R. A. (eds). Conservation Biology of Freshwater Turtles and Tortoises: A Compilation Project of the IUCN/SSC Tortoise and Freshwater Turtle Specialist Group. Chelonian Res. Monographs. p.042.041-042.049. http://doi.org/10.3854/crm.5.042.callirostris.v1.2010

9. BOCK, B.C.; PÁEZ, V.P.; DAZA, J.M. 2012. Trachemys callirostris (Gray 1856). En: PÁEZ, V.P.; MORALES-BETANCOURT, M.A.; LASSO, C.A.; CASTAÑO-MORA, O.V.; BOCK, B.C. (eds). V. Biología y Conservación de las Tortugas Continentales de Colombia. Ed. Recursos Hidrobiológicos y Pesqueros Continentales de Colombia; Instituto de Investigación de los Recursos Biológicos Alexander von Humboldt (Bogota). p.283-291

10. CONOVER, W.J. 1999. Practical nonparametric statistics. John Wiley \& Sons, Inc. (New York)

11. CORPORACIÓN AUTÓNOMA REGIONAL DEL CENTRO DE ANTIOQUIA, CORANTIOQUIA. 2008. Niveles de mercurio en sedimento, agua y tejido vivo "buchón, arroz, peces y cabello" en los humedales de la Mojana Sucreña.

12. CRUZ-ESQUIVEL, Á.; VILORIA-RIVAS, J.; MARRUGONEGRETE, J. 2017. Genetic damage in Rhinella marina populations in habitats affected by agriculture in the middle region of the Sinú River, Colombia. Environmental Science Pollution Research (Alemania). 24(35):27392-27401. https://doi.org/10.1007/s11356-017-0134-8

13. DE LA OSSA-V, J.; DE LA OSSA-LACAYO, A. 2018. Hunting, use and Trade of Chelonians in the South of Sucre, Colombia. Indian J. Science And Technology (India). 11(36). http://doi.org/10.17485/ijst/2018/v11i36/131571 
14. DIAZ, S.M.; PALMA, R.M.; MUÑOZ, M.N.; BECERRAARIAS, C.; FERNÁNDEZ NIÑO, J.A. 2020. Factors Associated with High Mercury Levels in Women and Girls from The Mojana Region, Colombia, 2013-2015. International Journal of Environmental Research and Public Health (Suiza). 17(6):1827. https://doi.org/10.3390/ ijerph17061827

15. DI RIENZO, J.A.; CASANOVES, F; BALZARINI, M.G.; GONZALEZ, L.; TABLADA, M.; ROBLEDO, C.W. 2011. InfoStat. Universidad Nacional de Córdoba, Córdoba, Argentina.

16. FOOD AND AGRICULTURE ORGANIZATION OF THE UNITED NATIONS, FAO. 2015. Codex Alimentarius. Normas Internacionales de los alimentos.Norma general para los contaminantes y las toxinas presentes en los alimentos y piensos. CODEX STAN 193-1995.

17. FRITZ, U.; STUCKAS, H.; VARGAS-RAMÍREZ, M.; HUNDSDÖRFER, A.K.; MARAN, J.; PÄCKERT, M. 2012. Molecular phylogeny of Central and South American slider turtles: implications for biogeography and systematics (Testudines: Emydidae: Trachemys). Journal of Zoological Systematics and Evolutionary Research (Alemania). 50(2):125-136. https://doi.org/10.1111/ j.1439-0469.2011.00647.x

18. GREEN, A.D.; BUHLMANN, K.A.; HAGEN, C.; ROMANEK, C.; GIBBONS, J.W. 2010. Mercury contamination in turtles and implications for human health. J. Environmental Health (Estados Unidos). 72(10):14-22.

19. HOLDRIDGE, L. 1967. Life zone ecology. Tropical Science Center. San Jose, Costa Rica. 206p.

20. HONG, Y.-S.; KIM, Y.-M.; LEE, K.-E. 2012. Methylmercury exposure and health effects. Journal of preventive medicine and public health (Corea del Sur). 45(6):353-363. http:// doi.org/10.3961/jpmph.2012.45.6.353

21. KRUSKAL, W.H.; WALLIS, W.A. 1952. Use of ranks in one-criterion variance analysis. Journal of the American statistical Association (Estados Unidos). 47(260):583-621. http://doi.org/10.1080/01621459.1952.10483441

22. LEGUIZAMO, C.; BONILLA, M.A. 2014. Reproducción de Trachemys callirostris callirostris (Emydidae) en ambientes generados por la minería en La Guajira, Colombia. Acta Biológica Colombiana (Colombia). 19(3):363-380. https:/ / doi.org/10.15446/abc.v19n3.41597

23. MARRUGO, J.; PINEDO-HERNÁNDEZ, J.; PATERNINAURIBE, R.; QUIROZ-AGUAS, L.; PACHECOFLOREZ, S. 2018. Distribución espacial y evaluación de la contaminación ambiental por mercurio en la región de la Mojana, Colombia. Revista MVZ Córdoba (Colombia). 23:7062-7075. https://doi.org/10.21897/rmvz.1481

24. MARRUGO-NEGRETE, J.; DURANGO-HERNANDEZ, J.; CALAO-RAMOS, C.; URANGO-CARDENAS, I.; DIEZ, S. 2019. Mercury levels and genotoxic effect in caimans from tropical ecosystems impacted by gold mining. Science of the Total Environment (Reino Unido). 664:899-907. http://doi.org/10.1016/j.scitotenv.2019.01.340

25. MARRUGO-NEGRETE, J.; VARGAS-LICONA, S.; RUIZGUZMÁN, J.A.; MARRUGO-MADRID, S.; BRAVO, A.G.; DÍEZ, S. 2020. Human health risk of methylmercury from fish consumption at the largest floodplain in Colombia. Environmental Research (Estados Unidos). 182:109050. https://doi.org/10.1016/j.envres.2019.109050

26. MELIDONE, R.; GIBSON, C.J. 2010. Necropsy techniques in reptiles Part 1: General introduction and Lizards and Crocodilians. Companion Animal (Reino Unido). 15(5):5358. http://doi.org/10.1111/j.2044-3862.2010.tb00488.x

27. MINISTERIO DE MINAS Y ENERGÍA-COLOMBIA, MINMINAS. 2014. Estudio de la cadena de mercurio en Colombia con enfasis en la actividad minera de oro. Bogotá, Colombia. 123p.

28. MORCILLO, P.; ESTEBAN, M.A.; CUESTA, A. 2016. Heavy metals produce toxicity, oxidative stress and apoptosis in the marine teleost fish SAF-1 cell line. Chemosphere (Reino Unido). 144:225-233. http://doi.org/10.1016/j. chemosphere.2015.08.020

29. PÁEZ-OSUNA, F.; CALDERON-CAMPUZANO, M.F.; SOTO-JIMENEZ, M.F.; RUELAS-INZUNZA, J. 2011. Mercury in blood and eggs of the sea turtle Lepidochelys olivacea from a nesting colony in Oaxaca, Mexico. Mar Pollut Bull (Reino Unido). 62(6):1320-1323. http://doi. org/10.1016/j.marpolbul.2011.04.004

30. PÁEZ, V.P.; MORALES BETANCOURT, M.A.; LASSO, C.A.; CASTAÑO MORA, O.V.; BOCK, B. 2012. Biología y conservación de las tortugas continentales de Colombia. Instituto de Investigación de Recursos Biológicos Alexander von Humboldt (IAvH). Bogotá, D. C., Colombia. 528p.

31. PARRA, M.P.; GUERRERO, M.N.M.; GARCÍA, O.P.; GÓMEZ, Y.O.; DÍAZ, S.M. 2019. Niños y adolescentes expuestos ambientalmente a mercurio, en diferentes municipios de Colombia. Revista Salud UIS (Colombia). 51(1):43-52.

32. ROCHA-ROMÁN, L.; OLIVERO-VERBEL, J.; CABALLERO-GALLARDO, K.R. 2018. Impacto de la minería del oro asociado con la contaminación por mercurio en suelo superficial de San Martín de Loba, sur de Bolívar (Colombia). Revista internacional de 
contaminación ambiental (México). 34(1):93-102. https:// doi.org/10.20937/RICA.2018.34.01.08

33. RUEDA-ALMONACID, J.V.; CARR, J.L.; MITTERMEIER, R.A.; RODRÍGUEZ-MAHECHA, J.V.; MAST, R.B.; VOGT, R.C.; RHODIN, A.G.; DE LA OSSAVELÁSQUEZ, J.; RUEDA, J.N.; MITTERMEIER, C.G. 2007. Las tortugas y los cocodrilianos de los países andinos del trópico. Editorial Panamericana y Formas e Impresos (Bogotá, D.C.). 270p.

34. SADIQ, M.; ZAIDI, T.; AL-MOHANA, H. 1991. Sample weight and digestion temperature as critical factors in mercury determination in fish. Archives of Environmental Contamination and Toxicology (Alemania). 47(3):335-341. https://doi.org/10.1007/BF01702191

35. SCHNEIDER, L.; EGGINS, S.; MAHER, W.; VOGT, R.C.; KRIKOWA, F.; KINSLEY, L.; EGGINS, S.M.; DA SILVEIRA, R. 2015. An evaluation of the use of reptile dermal scutes as a non-invasive method to monitor mercury concentrations in the environment. Chemosphere (Reino Unido). 119:163-170. https://doi.org/10.1016/j. chemosphere.2014.05.065
36. SMITH, P.N.; COBB, G.P.; GODARD-CODDING, C.; HOFF, D.; MCMURRY, S. T.; RAINWATER, T.R.; REYNOLDS, K.D. 2007. Contaminant exposure in terrestrial vertebrates. Environmental pollution (Reino Unido). 150(1):41-64. http://doi.org/10.1016/j.envpol.2007.06.009

37. SOUZA-ARAUJO, J.; GIARRIZZO, T.; LIMA, M.O. 2015. Mercury concentration in different tissues of Podocnemis unifilis (Troschel, 1848) (Podocnemididae: Testudines) from the lower Xingu River - Amazonian, Brazil. Brazilian Journal of Biology (Brasil). 75(3 Supl.1):106-111. http:// dx.doi.org/10.1590/1519-6984.01514BM

38. ZAPATA, L.M.; BOCK, B.C.; OROZCO, L.Y.; PALACIO, J.A. 2016. Application of the micronucleus test and comet assay in Trachemys callirostris erythrocytes as a model for in situ genotoxic monitoring. Ecotoxicology and environmental safety (Estados Unidos). 127:108-116. http://doi. org/10.1016/j.ecoenv.2016.01.016

39. ZAPATA, L.M.; BOCK, B.C.; PALACIO, J.A. 2014. Mercury concentrations in tissues of Colombian Slider turtles, Trachemys callirostris, from northern Colombia. Bulletin of environmental contamination toxicology (Alemania). 92(5):562-566. https://doi.org/10.1007/s00128-014-11985 